\title{
Application of Smart Fiber Optic Sensor Technology in Feature Extraction, Recognition, and Detection
}

\author{
Yuxiang Gao iD and Qixin Cai \\ State Grid Jiangsu Electric Power Co., Ltd., Marketing Service Center, Nanjing 210019, China \\ Correspondence should be addressed to Yuxiang Gao; nuaamrg@qq.com
}

Received 29 October 2021; Revised 13 December 2021; Accepted 30 December 2021; Published 8 February 2022

Academic Editor: Haibin Lv

Copyright ( 2022 Yuxiang Gao and Qixin Cai. This is an open access article distributed under the Creative Commons Attribution License, which permits unrestricted use, distribution, and reproduction in any medium, provided the original work is properly cited.

To improve the signal recognition effect of the security system, this paper studies the security system based on intelligent Fiber Optic Sensor (FOS) technology. Firstly, the research background of intelligent FOS is introduced, and its current situation in feature extraction, recognition, and detection is introduced. Secondly, the double Mach-Zehnder (M-Z) Optical Fiber- (OF-) based interferometer model is implemented, and the Wavelet Analysis (WA) theory is introduced to analyze the characteristic threshold and Frequency (F) characteristics of intrusion signal. Finally, the distributed intelligent FOS-based perimeter security system is constructed, and an empirical study is conducted to verify its performance. The results show that the intruder knocking-induced signal $\mathrm{F}$, intruder climbing-induced signal $\mathrm{F}$, noiseless environment-induced signal $\mathrm{F}$, and rainy environment-induced signal $\mathrm{F}$ are $0-250 \mathrm{kHz}, 0-25 \mathrm{kHz}, 0-1.5 \mathrm{kHz}$, and $0-3.5 \mathrm{kHz}$, respectively; in all the four cases, excellent results have been obtained after wavelet threshold denoising. Meanwhile, the received signal is decomposed into seven layers through multiscale WA theory. The signal feature classification is based on WA and takes variance as the representation, and the clear classification results are obtained; when the False Alarm Rate $(\mathrm{FAR})=1 \%$, the detection probability of the proposed system is about $99 \%$, while the detection probability of the traditional system is about $3 \%$. The reference arm and sensing arm of the distributed OF-based perimeter security system can be laid in the same optical cable. Therefore, the designed wavelet threshold filtering method is feasible, and the detection probability of the designed WA-based system is higher than that of the traditional security system. The research content provides a reference for the development of intelligent FOS technology in the field of security.

\section{Introduction}

The development of optical communication and Optical Fiber (OF) drawing techniques has given birth to the novel Fiber Optic Sensor (FOS) technology. In particular, FOS technology is proposed in the late 1970s and since then has developed rapidly [1]. Compared with traditional sensors, FOS is more advantageous, featuring long-distance detection, anticombustion, antiexplosion, and corrosion resistance, as well as high sensitivity, antielectromagnetic interference, and continuously distributed detection [2]. The FOS can complete the intrusion events-oriented Pattern Recognition (PR) by collecting the information of vibration points and using Artificial Intelligence (AI) and Data Mining (DM) technologies [3]. FOS can be divided into distributed
FOS and quasidistributed FOS. The OF in the distributed FOS technology functions both as a sensor and light source transmission medium; it can inspect the whole area but also realizes fuzzy identification of the intrusion events in the detection area [4]. According to the nature of the signal, FOS technology can be divided into Raman effect-enabled sensing technology, interference effect-enabled sensing technology, Brillouin effect-enabled sensing technology, forward transmission mode coupling-enabled sensing technology, and backward Rayleigh scattering-enabled sensing technology. Distributed intelligent FOS technology is mostly used in the application of OF-based Alarm Systems (AS) outside China [5]. The United States was the first to apply OF technology. In 1992, FOS technology was applied to AS with satisfactory results [6]. Australia developed a 
monitoring and protection system for long-distance oil pipelines and OF lines at the end of the twentieth century. The system adopted vibration and mobile sensors. The whole system encompassed photoelectric modulation and demodulation equipment, sensitive Optical Cable (OC), and computer-based automatic identification algorithm software, with a relatively small False Alarm Rate (FAR) [7]. In the early twenty-first century, the United States developed the FOS technology for monitoring energy pipelines, which had become the predecessor of intelligent FOS technology. At present, the intelligent FOS technology in Western developed countries is mainly used for qualitative alarm [8].

The research on FOS technology in China has seen a late start and is still in the stage of scheme exploration and theoretical research [9]. In the early twentieth century, the Chinese Academy of Sciences developed a vibration-oriented FOS system based on the Michelson interferometer optical path scheme. The system presented a wide detection range even though lacked a positioning function [10]. In 2008, Fudan University in Shanghai developed a vibrationoriented FOS system with a positioning function [11]. In 2010, China's distributed FOS technology has been applied to the damage monitoring of submarine oil pipelines [12]. With the development of Artificial Intelligence (AI) technology and Internet of Things (IoT) technology, intelligent FOS technology has also been applied to IoT-related research [13]. In particular, the primary role of intelligent FOS technology in the field of IoT is to rely on its feature extraction, identification, and detection to detect intrusion events in the IoT system, as well as indoor positioning of intelligent mobile terminals [14]. The high sensitivity of FOS technology will bring high FAR. Therefore, in practical application, it is necessary to use the signal extraction and identification of vibration points of FOS to reduce the system FAR [15]. At present, the commonly used Feature Extraction (FE) methods of intelligent FOS include the Support Vector Machine (SVM), Convolution Neural Network (CNN) method, data denoising, and compression classifier method. For example, Min et al. (2021) found that intelligent FOS played an important role in marine environment detection [16]. Bado et al. (2021) studied the postprocessing algorithm of distributed FOS in structural health detection, which improved the feasibility of distributed FOS [17]. Du et al. (2021) applied FOS technology to coal seam structure and found that fiber Bragg grating and distributed OF method could monitor the stability of mining overburden [18]. In the previous research, the research on light intelligent sensor technology mainly focused on the application of AS and equipment detection, and the research on the accuracy of intrusion AS was mostly theoretical.

Under the background of IoT, this paper applies intelligent FOS technology to the research of feature extraction, recognition, and detection of intrusion in the intelligent security system. Firstly, the research background of FOS technology is introduced. Secondly, the problem is studied through information collection, feature extraction, classification, and recognition technology. Finally, the simulation experiment is designed and the experimental results are analyzed. The innovation point is to propose a distributed
OF intrusion detection technology based on the principle of Mach-Zehnder (M-Z) OF-based interferometer, which improves the problem of high FAR and false-positive alarm rate of intrusion detection system at the present stage. The research content provides an improvement direction for the development of intelligent FOS technology in the field of IoT and lays a foundation for the development of intelligent FOS technology in fault detection.

\section{Research on Application Approach of Smart FOS}

2.1. Selection of FOS. So far, smart FOS has developed into microbending FOS, Optical Time Domain Reflector (OTDR) FOS, Sagnac interferometric vibration FOS, and $\mathrm{M}-\mathrm{Z}$ interferometric vibration FOS [19]. Microbending FOS is an all FOS constructed based on the principle of optical carrier intensity change in the cladding from microbending deformation with low sensitivity [20]. OTDR vibration FOS is constructed based on the optical time-domain reflection principle with low sensitivity [21]. Sagnac interferometric vibration FOS is based on the Sagnac interferometer, which has high sensitivity but cannot sense the vibration phase modulation, and the Signal-to-Noise Ratio (SNR) cannot be improved by averaging technique [22]. The $\mathrm{M}-\mathrm{Z}$ interferometric vibration FOS is based on the $\mathrm{M}-\mathrm{Z}$ interferometric principle; the ambient vibration on OC can be easily monitored. The specific structure of the $\mathrm{M}-\mathrm{Z}$ interferometric vibration FOS is shown in Figure 1.

Figure 1 illustrates that the coherent beam starts from the light source, is divided into two beams at the coupler 1, and propagates into the reference arm and the sensing arm. Finally, it interferes at the position of coupler 2 and is received by the detector. Once the external vibration acts on the signal arm, the phase of light wave in the signal arm will be modulated, and a phase difference will be formed between the reference arm and the signal arm. Then, the interference light intensity will be demodulated, and finally, the Phase Modulation (PM) signal generated by the original vibration is obtained [23].

$\mathrm{M}-\mathrm{Z}$ interferometric FOS has high sensitivity and is independent of the action point and Frequency (F) of PM [24]. M-Z interferometric FOS is prone to relative drift and amplitude fading because the arm lengths of the reference arm and signal arm cannot be consistent [25]. The modem technique can be used to solve the relative drift problem. $\mathrm{M}-\mathrm{Z}$ interferometric FOS is widely used in OF accelerometers and OF hydrophones because of its high sensitivity and consistent sensitivity on the signal arm [26]. Based on its high sensitivity, M-Z interferometric FOS is used as the research basis here.

\subsection{Design of Feature Extraction and Recognition Monitoring} Scheme. This research is set against the background of smart SPS, so features of the detection system signal are extracted for research; the features of the detection signal are understood and analyzed. Malicious behaviors, such as crossing over the OC, will give an instantaneous stimulation 


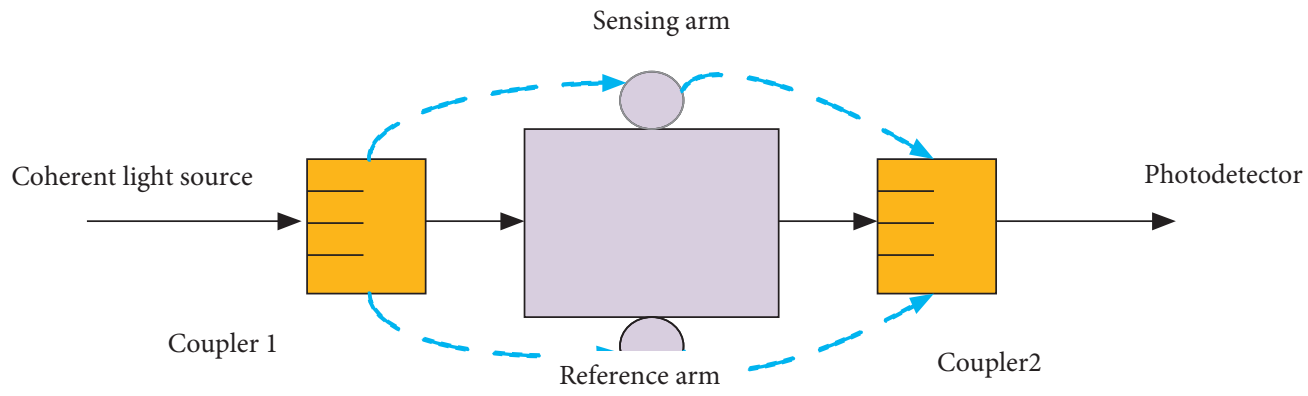

FIgURE 1: M-Z interferometric vibration FOS structure.

to the OF and form an exciting vibration signal, which is characterized by gradual attenuation over time [27].

OF affected by the vibration wave will undergo elastic deformation, resulting in the change of the phase of the propagating light wave [28]. When the OF is free from an external force, the calculation of outgoing light wave reads:

$$
E_{2}=E_{1} \exp \left(\frac{j 2 p n L}{c}\right)
$$

In equation (1), the length of the OF is $L$, the incident light wave is $E_{1}$, and when the $\mathrm{OF}$ is not subjected to external force, the outgoing light wave is $E_{2}, c$ represents the speed of light, and $n$ denotes the refractive index of the OF. $p$ and $j$ are the photoelastic coefficient and the Constant Phase Element (CPE), respectively.

The additional phase under the action of external force is set as $\Delta \delta$. The following equation shows the outgoing light wave at this time.

$$
E_{2}=E_{1} \exp \left[j\left(\frac{2 p n L}{\lambda}+\Delta \delta\right)\right]
$$

In equation (2), $\lambda$ represents the wavelength of light; the specific expression of $\Delta \delta$ is shown in the following equation:

$$
\Delta \delta=b L \frac{\Delta L}{L}+L \frac{\partial b}{\partial n} \Delta n+L \frac{\partial b}{\partial a} \Delta a .
$$

In equation (3), $b$ represents the propagation constant of the light waves in the OF, and $a$ indicates the radius of the OF.

After the introduction of elasticity theory, the expression of the output light wave of OF subjected to vibration is shown in the following equation:

$$
E_{2}=E_{1} \exp \left\{j\left[\frac{2 p n L}{\lambda}+B \cdot f\left(t-\frac{z}{c} n\right)\right]\right\} \text {. }
$$

In equation (4), $p$ represents the pressure on the OF, $B$ is the constant, and $z n / c$ stands for a certain moment of the pressure on the OF. $c, z$, and $f$ represent the constant, phase change difference, and the vibration force, respectively.

Next, the mathematical model of the M-Z OF-based interferometer is implemented. Based on Figure 1, the components of the M-Z OF-based interferometer include coupler, light source, and detector. When the external force acts on the $M-Z$ interferometer, the light wave received by the detector passing through the reference arm and the sensing arm is shown in the following equations:

$$
\begin{aligned}
& E_{g}=\frac{\sqrt{2}}{2} E_{1} \exp \left\{j\left[\frac{2 \pi n(L+\Delta L)}{\lambda}+B \cdot f(t-\tau)\right]\right\}, \\
& E_{k}=\frac{\sqrt{2}}{2} E_{1} \exp \left[j\left(\frac{2 \pi n L}{\lambda}+\pi\right)\right] .
\end{aligned}
$$

In equations (5) and (6), $\tau$ represents the time when the light wave reaches the detector; $B f(t-\tau)$ denotes the PM of the light wave by the external force. $L$ is the length of the reference arm; $L+\Delta L$ means the length of the sensing arm.

The expression of the final interference output $I$ is shown in the following equation:

$$
I=\frac{1}{4} E_{1}^{2}\left\{1+\cos \left[\Delta \varphi_{L}+B f(t-\tau)\right\} .\right.
$$

In equation (7), $I$ denotes the final light intensity interference output. $\Delta \varphi_{L}=2 \pi n L / \lambda-\pi$ and $\Delta \varphi_{L}$ indicate the initial phase difference between the signal arm and the reference arm, respectively.

Next, the mathematical model of the optical path of FOS perimeter SPS is implemented. Based on the idea of making full use of the information in the $M-Z$ interferometer, an interferometric FOS system with a dual $\mathrm{M}-\mathrm{Z}$ structure is constructed. The structure diagram of the system is shown in Figure 2.

Figure 2 indicates that the interferometric FOS system with a dual $\mathrm{M}-\mathrm{Z}$ structure is composed of forwarding and backward parts. The detector PD2 receives the forward interference signal transmitted through the guiding OF L4, and the detector PD1 receives the backward interference signal transmitted through the guiding of L3. C1, C2, and C3 are $3 \mathrm{~dB}$ couplers.

The distribution diagram in practical application is shown in Figure 3.

Figure 3 reveals that the sensing fiber and guiding fiber are wrapped in the same OC [29]. Based on the previous content, the final mathematical model of interferometric FOS with dual M-Z structure is given here, as shown in the following equations:

$$
\begin{aligned}
& I_{1}=\frac{1}{8} I_{0}+\frac{1}{8} I_{0} \cos \left[\varphi_{0}+(1+S) \Delta \varphi\left(t-\tau_{1}\right)\right], \\
& I_{1}=\frac{1}{8} I_{0}+\frac{1}{8} I_{0} \cos \left[\varphi_{0}+(1+S) \Delta \varphi\left(t-\tau_{2}\right)\right] .
\end{aligned}
$$




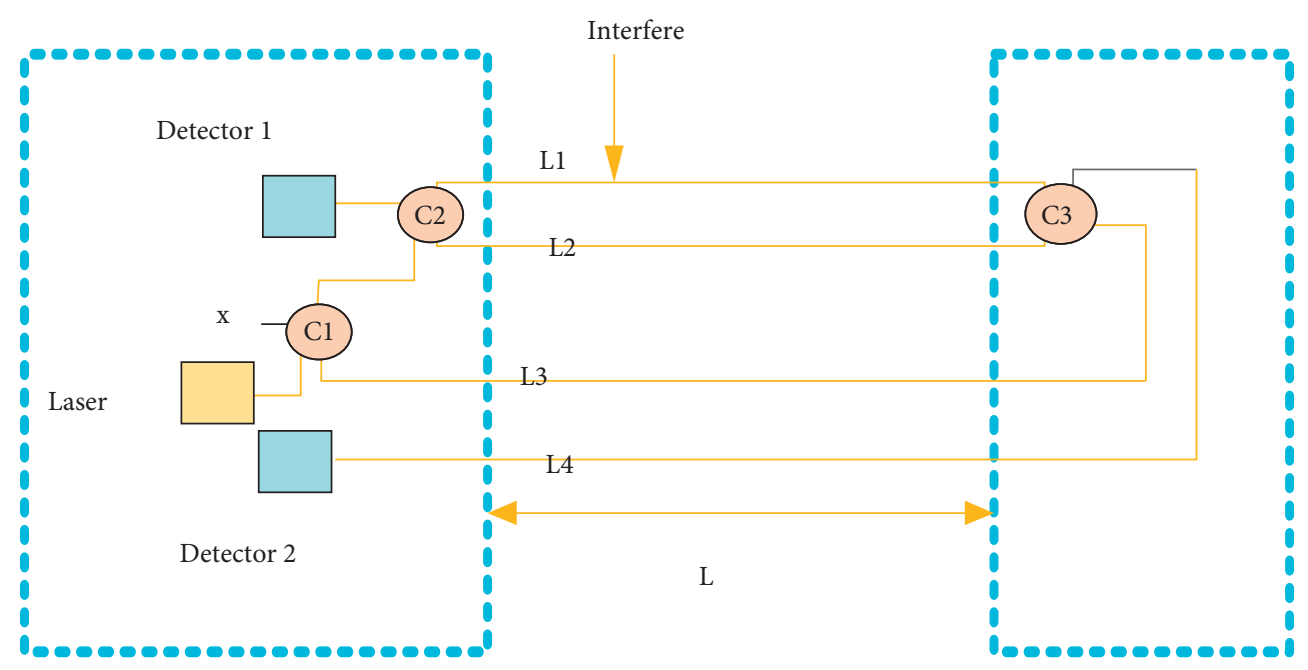

FIgURE 2: Structure diagram of the interferometric FOS system with dual M-Z structure.

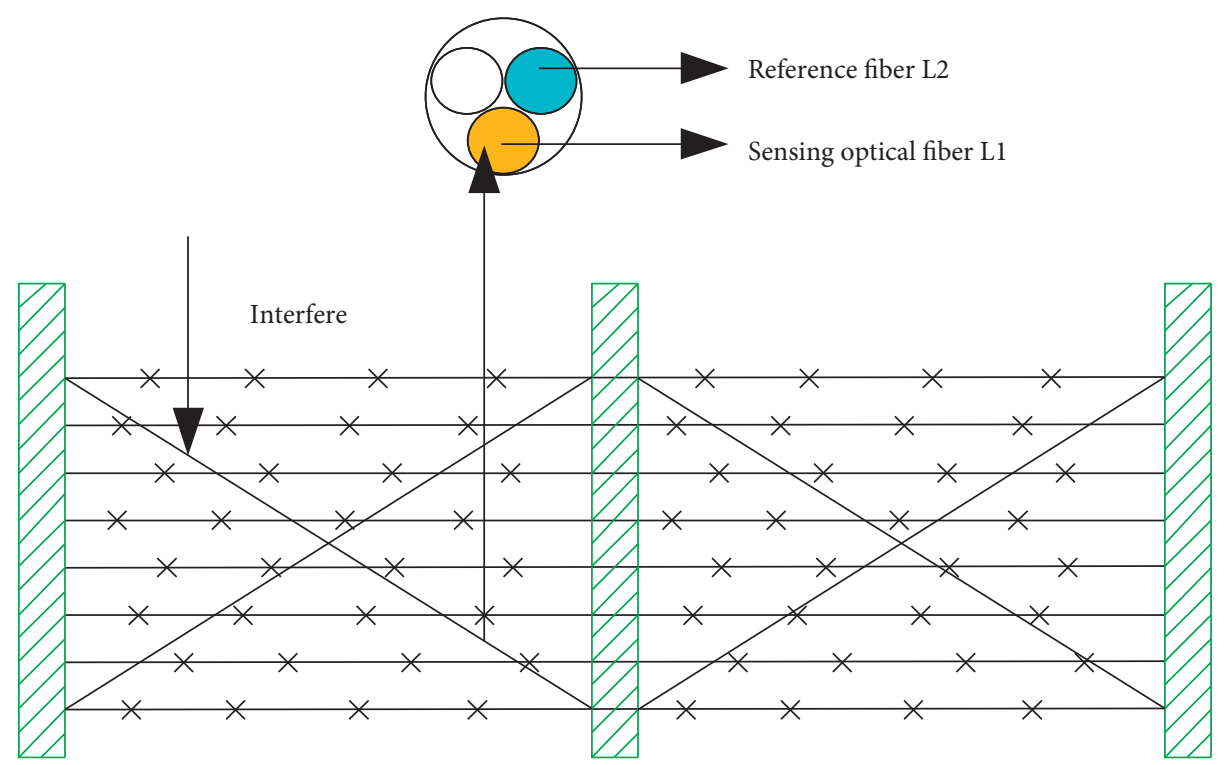

Figure 3: L1 and L2 distribution of sensing fiber.

In equations (8) and (9), $S$ is a constant, $I$ represents the interference light intensity, $\varphi_{0}$ denotes the initial phase, and the $\mathrm{M}-\mathrm{Z}$ interferometer's reference and sensing arms do not need to be separately laid.

The research experiment for signal feature extraction and analysis of FOS SPS is set on a border defense area. The OC length of the OF vibration test is $58 \mathrm{~km}$. The real vibration signal is analyzed combined with the nonstationary signal processing theory. The experimental conditions are configured with unchanged circuit gain, interferometer arm length difference, and light source power. The intrusion contacts include knocking and climbing over the OC. Meanwhile, the signal characteristics of the ambient noise, including no ambient noise and rainy ambient noise, and the $\mathrm{F}$ of the corresponding vibration signal are collected. The experimental instrument is provided by the Metrological Production Automation Workshop. The experimental data set is collected from the OF noise experimental data in the study area from August 7, 2021, to August 14, 2021. The specific experimental scheme is given in Section 3.5.

2.3. Performance Test of FOS SPS. In the signal detection of distributed FOS SPS, the signal processing model is the best detection problem of Gaussian signal under the background of Gaussian noise. The actual best system is an approximate average power detector. The output SNR is set as follows:

$$
\left(\frac{F}{N}\right)_{r}=\frac{\sigma_{F}^{2}(r)}{\sigma_{0}^{2}(r)}
$$

In equation (10), $r, F$, and $N$ represent the process, the signal, and the noise, respectively; $\sigma_{F}^{2}$ denotes the last energy detector value, and $\sigma_{0}^{2}$ indicates the initial energy detector value. The output SNR of the optical receiving system under the conditions of average detector and Gaussian is shown in the following equation: 


$$
\left(\frac{F}{N}\right)_{z}=T W_{y}\left(\frac{F}{N}\right)_{x}^{2} .
$$

In equation (11), $z, x$, and $y$ represent the output process, the input process, and an instant of the process, respectively; $W$ stands for the output power. The output Signal-to-Noise Ratio (SNR) of the square detector is proportional to the square of the input SNR.

The detection performance of the system is comprehensively evaluated through SNR, FAR, and detection rate. The curve of these three indexes is called the Receiver Operation Curve (ROC) [30]. Here, the output test statistic $z$ is used as a Gaussian variable, and the threshold $k$ is used. The expression of transformed FAR and detection rate is shown in the following equations:

$$
\begin{aligned}
& P_{1}=1-G K_{n}, \\
& P_{2}=1-G\left(K_{n}-\left(\frac{F}{N}\right)_{z}^{1 / 2}\right.
\end{aligned}
$$

In equations (12) and (13), $P_{1}$ represents FAR, $P_{2}$ indicates detection rate, $G$ is Gaussian distribution function, $K_{n}$ is the normalized threshold, and equations (12) and (13) are the basis of the ROC.

2.4. Intrusion Signal Identification and Detection of SPS. Wavelet Analysis (WA) is used for feature recognition and classification detection of intrusion signals. WA is a branch of Applied Mathematics based on harmonic analysis theory. Wavelet transform has an excellent performance in analyzing signals [31]. The multiresolution analysis is one of the important concepts in WA. Multiresolution analysis can provide a framework for constructing wavelets and a fast algorithm for function reconstruction and decomposition [32]. WA has been widely used in the multiresolution analysis. WA can project the signal onto the wavelet function subspace, obtain the expansion of the signal on different scales, finally, obtain the signal characteristics of different $\mathrm{F}$ bands, and retain the time-domain characteristics of the signal [33].

Here, if the energy corresponding to the high-F signal level is EI, the energy of each F band signal can be expressed as in the following equation under the WA scale:

$$
E_{i}=\frac{1}{n} \sum_{k=1}^{n}\left|d^{j}(k)\right|^{2}
$$

In equation (14), $d j(k)$ is the value of each discrete point on the $j$ th scale of WA. $d$ represents the number of layers, $k$ stands for the number of scattered points, and $n$ refers to the number of scattered points.

If $M$ is the eigenvector of energy $E i$, the expression of $M$ reads

$$
M=\left[E_{1}, E_{2}, \ldots, E_{i} \ldots\right] .
$$

In equation (15), $i=1,2, \ldots, I$.
Different eigenvalues are obtained by experimental statistics. The expression of eigenvalues of different test times is shownas follows:

$$
e_{i}=\frac{1}{n} \sum_{k=1}^{n} e_{i k}
$$

In equation (16), $k=1,2, \ldots, n$, where $n$ is the number of experiments.

Wavelet analyses are used to decompose the time-domain waveform into different $\mathrm{F}$ bands to obtain time-domain signals, and the time-domain waveform is used for energy statistics. The difference of characteristic distribution of different vibration signals is the basis for distinguishing the types of vibration signals.

Wavelet denoising based on WA is one of the main denoising methods. Wavelet denoising has the characteristics of low entropy, multiresolution, diversity, selectivity, and decorrelation. The wavelet threshold shrinkage denoising method is the best for Gaussian denoise. The wavelet transform can concentrate the signal energy with fewer wavelet coefficients and then expand on the wavelet coefficients. The amplitude of the noise after wavelet decomposition is less than the wavelet coefficients of the signal. The wavelet coefficients of most noise are reduced to 0 by setting the threshold, and the wavelet coefficients of the signal are retained. This way, noise is eliminated. The basis of the wavelet threshold denoising algorithm is that when the noisy signal is decomposed, the assignment of wavelet transforms coefficient of the signal increases while the amplitude of the high-F wavelet decomposition coefficient decreases.

The wavelet threshold denoising algorithm first selects threshold parameters, threshold function, wavelet transform method, and wavelet basis. Common threshold parameters include the VisuShrink threshold, SureShrink threshold, and Minimax threshold. SureShrink threshold and Minimax threshold apply to a few high-F noisy signals. Therefore, the general VisuShrink threshold is selected. The specific expression is shown as follows:

$$
H=\xi \sqrt{2 \ln N} .
$$

In equation (17), $H$ represents the threshold, $\xi$ denotes the noise standard deviation, and $N$ is the signal length. In practical application, the noise standard deviation is obtained by decomposing the first layer of wavelet coefficients.

Commonly, threshold parameters are set by the hard threshold method or soft threshold method, both having advantages and disadvantages. This paper combines the hard threshold method and the soft threshold method. The designed threshold denoising algorithm is described as follows: first, the orthogonal wavelet transform of the noisy signal is calculated, then the nonlinear threshold approach is used to process wavelet coefficients, and finally, the inverse wavelet transform is carried out. The noise standard deviation in a practical application generally needs to be estimated. Through comparative analysis of various methods, a method suitable for practical problems is proposed. The specific steps are as follows: 
(1) Estimating noise standard deviation

$$
\xi_{n}=\frac{1}{0.6745} \cdot \frac{1}{N} \cdot \sum_{k=1}^{N}\left|w_{k}^{i}\right| .
$$

(2) In equation (18), $N$ is the number of wavelet coefficients, $w_{k}^{i}$ means the wavelet coefficients of the $i$ th layer, and the noise standard deviation is obtained by the MATLAB function. The function is $R n=$ wnoisest $(v, l, i)$, where $l$ stands for the length vector of the coefficients of each layer, $v$ indicates the wavelet decomposition coefficient, and $i$ represents the $i$ th layer.

(3) Threshold selection.

(4) The Birge2Massart algorithm, which has achieved good results in practical application, is used. The MATLAB function THR $=$ wbmpen $(v, l, R n, A)$ is adopted, where $A$ is generally taken as 2 , indicating the penalty coefficient.

(5) Processing threshold.

(6) Through steps 1 and 2, $i$ thresholds are obtained, all of which are global thresholds. Finally, the maximum threshold is selected as the global threshold for final noise reduction.

(7) Further, the results of wavelet threshold denoising in the case of ambient noise and climbing-over-OC behavior are presented to show the effect of wavelet threshold denoising.

2.5. Signal Recognition Application of Smart FOS in SPS. Based on previous sections, this section uses the wavelet multiscale analysis method to detect and identify the intrusion signal in the SPS. The experimental machine is the prototype of the distributed OF perimeter SPS, which is provided by the Measurement and Production Automation Workshop of Shaanxi Province. The research object is a border area. The terrain differs greatly over the research area, and the precipitation is heavy. The border patrol road and barbed wire are installed. The barbed wire is supported by cement columns every $5 \mathrm{~m}$, with a height of $2.5 \mathrm{~m}$. The patrol road and the barbed wire are distanced uncertainly. The main intrusion is to climb over the barbed wire. The actual test scheme is as follows: $28 \mathrm{~km}$ OC is laid, both arms of $\mathrm{M}-\mathrm{Z}$ interferometer are composed of $26 \mathrm{~km}$ single-mode OC, the total length of loop is $56 \mathrm{~km}, 3 \mathrm{~dB}$ single-mode coupler is selected as coupler, InGaAs photodetector is selected as the detector, and the working power is $300 \mu \mathrm{W}$. The wavelength is $1,550 \mathrm{~nm}$, the acquisition card is PCI-9812, the resolution is 12 bits, and the maximum acquisition rate is $20 \mathrm{MHz}$. The specific layout scheme is shown in Figure 4.

Figure 4 implies that two sections with a length of $13 \mathrm{~km}$ are fixed on the barbed wire, and an OC with a length of $2 \mathrm{~km}$ is submerged underground as a guiding OC. The experimental instruments, photoelectric module, and circuit module are provided by the Metrological Production Automation Workshop. In Figure 4, A, C, and D are $3 \mathrm{~dB}$ single-mode couplers, and $\mathrm{B}$ is the sensing cable.
Based on Figure 4, the energy feature extraction and recognition of noise signals are analyzed; under rain, noisefree, climbing-over-the-OC behavior, and knocking-the-OC behavior, and the feature vector extraction results are classified and recognized. Finally, the performance of the system is tested.

\section{Analysis of Application Results of Smart FOS Technology in SPS}

3.1. Intrusion Signal Features and Noise Results of SPS. The F-domain waveforms of intruders knocking, climbing over the barbed wire, noise-free ambiance, and rainy environment are shown in Figure 5.

Figure 5 implies that in these four cases, the $\mathrm{F}$ of the signal under the intruder-knocking behavior, the intruderclimbing behavior, in the noiseless environment, and the rainy environment is $0-250 \mathrm{kHz}, 0-25 \mathrm{kHz}, 0-1.5 \mathrm{kHz}$, and $0-3.5 \mathrm{kHz}$, respectively. These data are the basis of signal feature distribution in this experiment. The following section will present the final feature distribution classification results based on the signal feature distribution of these four cases combined with the previous content. The waveforms obtained under the four environments of intruder knocking, climbing-over-the-OC, no noise, and rain are basically consistent with the shape of $\mathrm{F}$ domain waveforms in previous studies, and they also accord with the law of noise.

After wavelet threshold denoising, the feature analysis diagram of the signal is shown in Figure 6. Due to the space relationship, the denoising analysis of ambient noise is taken as an example.

Figure 6 demonstrates that the ambient noise has achieved good results after wavelet threshold denoising. Obviously, the selected wavelet threshold filtering method is highly feasible. Due to space limitations, the denoising results of the other three cases are simply described in the text; the signal under intruder-knocking, intruder-climbing, and in a rainy environment has good denoising effects. Thus, the proposed method is suitable for the four actual cases. Meanwhile, the specific trend of the graph implies that the effect of the proposed wavelet threshold denoising technology is better.

3.2. Feature Extraction and Recognition Test Results and System Performance Verification. Based on the previous content, the feature vector extraction results are given here. The signal energy is divided into 1-7 scales, and the variance represents the energy. The final classification results are shown in Table 1.

Table 1 suggests that the received signal is decomposed into seven layers through wavelet multiscale analysis theory. The signal feature classification is based on WA and characterized by variance. Finally, the classification results of signal features under four actual cases studied are obtained. In practical application, Table 1 can be referred to. The detection rate of the system can be greatly improved using clearly distinguished events and eigenvector distribution. Xu et al. (2020) found that feature classification was very 


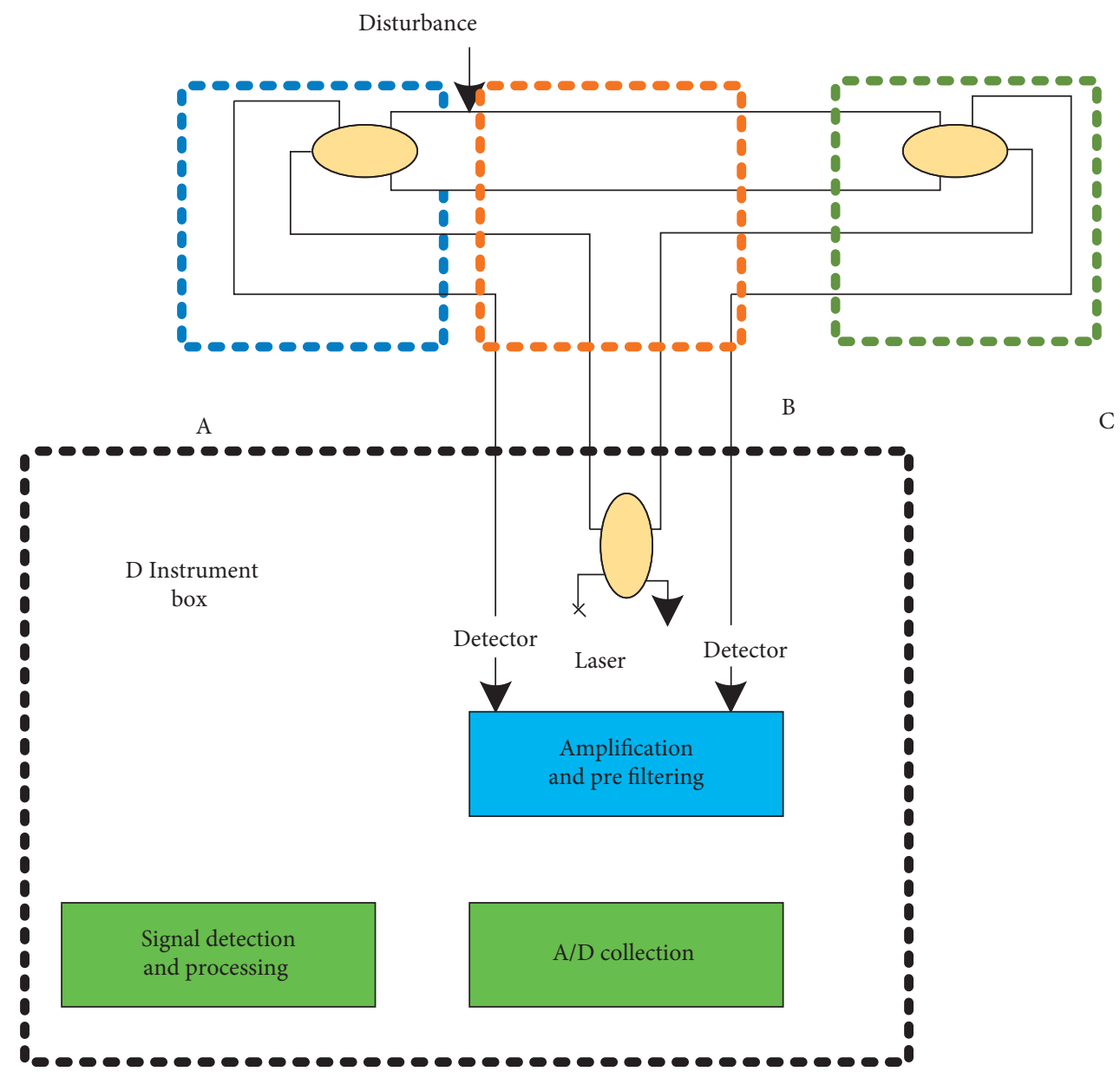

FIGURE 4: SPS layout.

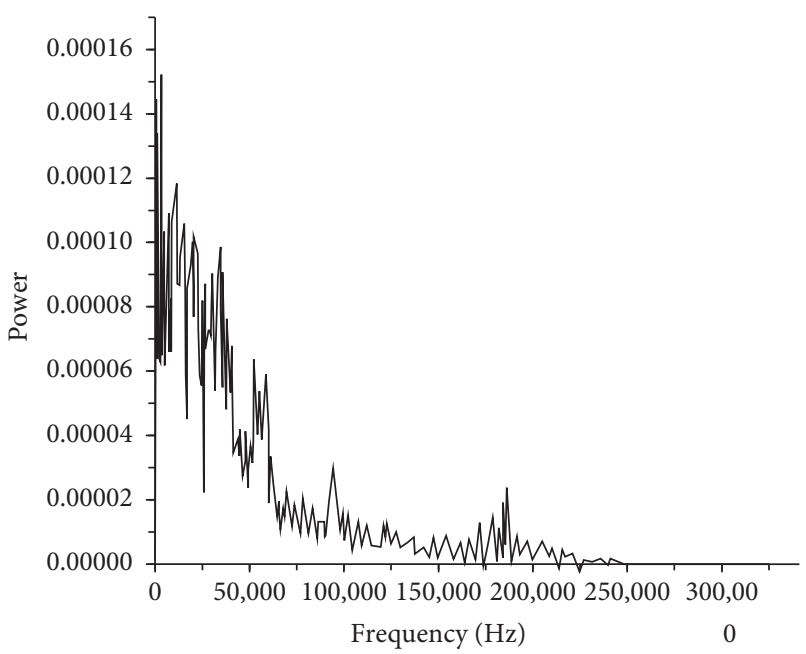

(a)

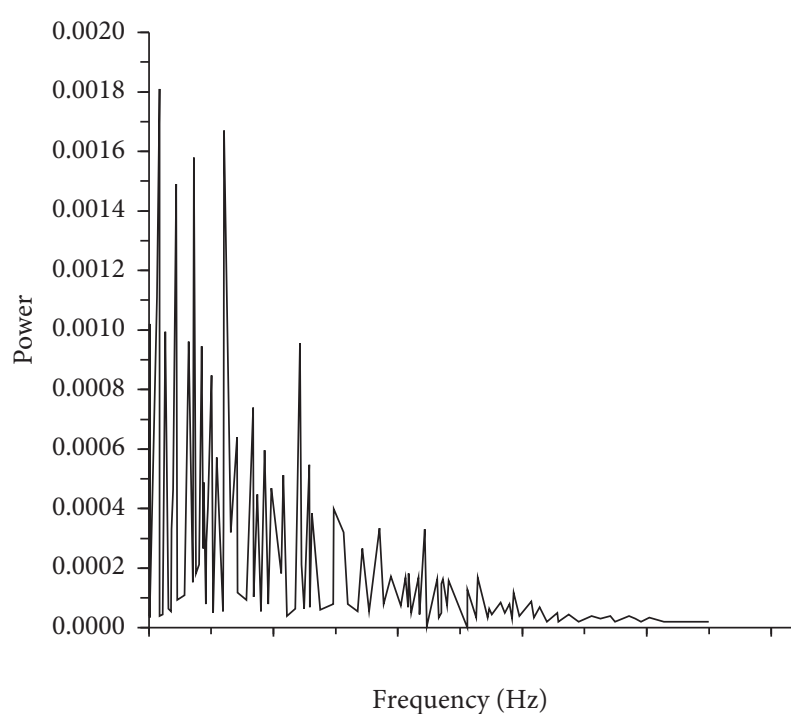

(b)

Figure 5: Continued. 


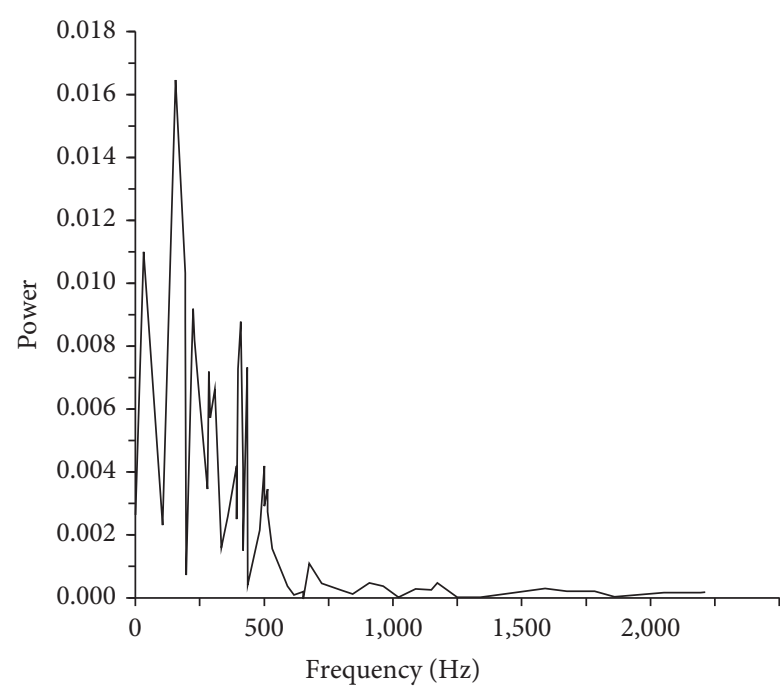

(c)

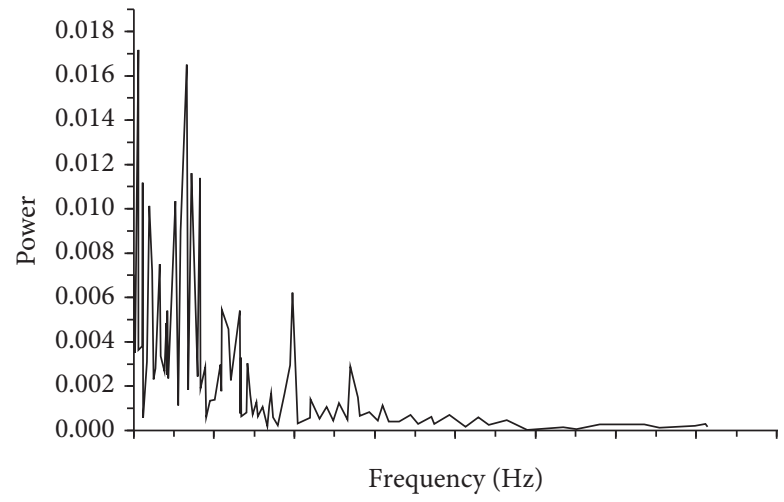

(d)

Figure 5: (a) The F domain waveform of the signal under the intruder-knocking behavior, (b) the F domain waveform of the signal under the intruder-climbing behavior, (c) the F domain waveform of the signal in the noise-free ambiance, and (d) the F domain waveform of the signal in the rainy environment.

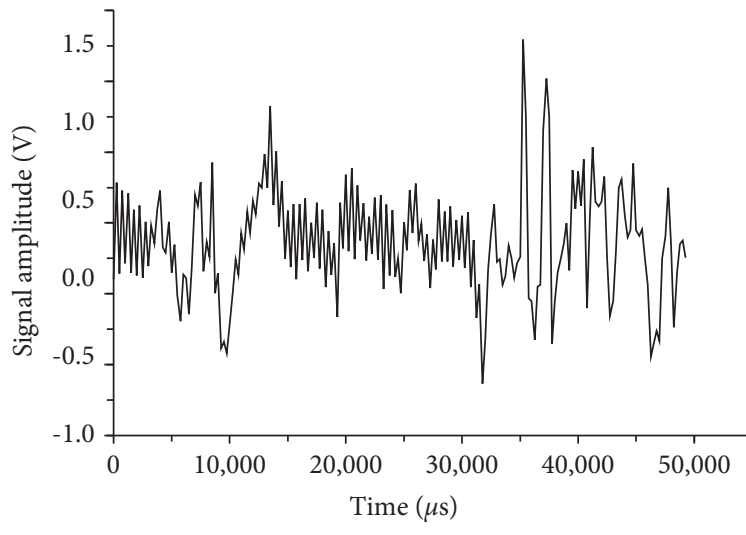

(a)

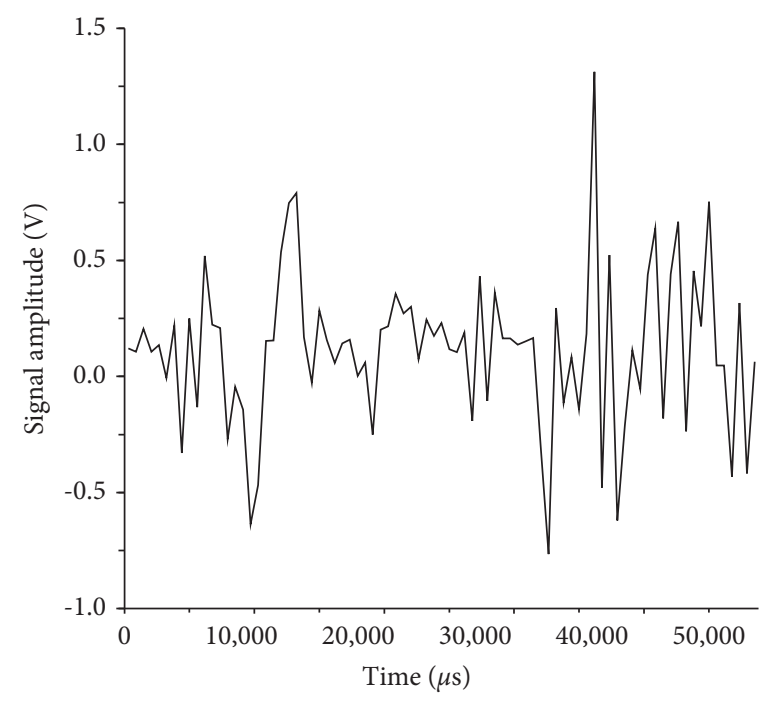

(b)

FiguRE 6: Wavelet threshold denoising results of ambient noise. (a) The signal before wavelet threshold denoising and (b) the signal after denoising.

important for machine learning and system prediction [34]. Hence, the content of Table 1 lays a data foundation for the improvement of system detection probability and the reduction of FAR, which is consistent with the research conclusions of the literature.

Finally, the detection rate of the proposed wavelet energy detector is compared with that of the traditional energy detector, and the detection rate under $1 \%$ FAR is taken as the comparison benchmark. The results are shown in Figure 7.

Figure 7 implies that the detection rate of the system based on the proposed WA is higher than that of the traditional SPS. When the FAR is $1 \%$, the detection rate of the proposed system is about $99 \%$, while the detection rate of the traditional system is about $3 \%$. Meanwhile, at $1 \%$ FAR, the detection rate of the proposed system still increases slowly with the increase of the FAR. Thus, the signal detection performance of the system based on the proposed WA is excellent. The detection probability of traditional energy detectors is greatly affected by ambient factors, such as OC vibration. Wavelet energy detector has better performance because it can effectively identify various excitations causing $\mathrm{OC}$ vibration. 
TABLE 1: Signal feature energy classification results.

\begin{tabular}{|c|c|}
\hline Specific case & Classification judgment basis \\
\hline The intruder knocks on the OC & $\begin{array}{c}60 \%>c^{7} \text { scale energy }>45 \%, 40 \%>d^{7} \text { scale energy }>30 \%, 20 \%>d^{6} \text { scale energy }>10 \%, 3 \%>\text { other scale } \\
\text { energy }\end{array}$ \\
\hline $\begin{array}{l}\text { The intruder climbs over the } \\
\text { OC }\end{array}$ & $\begin{array}{c}75 \%>c^{7} \text { scale energy }>60 \%, 37 \%>d^{7} \text { scale energy }>21 \%, 12 \%>d^{6} \text { scale energy }>5 \%, 2 \%>\text { other scale } \\
\text { energy }\end{array}$ \\
\hline No obvious ambient noise & $c^{7}$ scale energy $>99 \%$ \\
\hline Rainy conditions & $95 \%>c^{7}$ scale energy $>90 \%, d^{7}$ scale energy $>3 \%, 2 \%>$ other scale energy \\
\hline
\end{tabular}

Note. $c$ represents the low F signal level, $d$ denotes the high F signal level, and the superscript number stands for the level position.

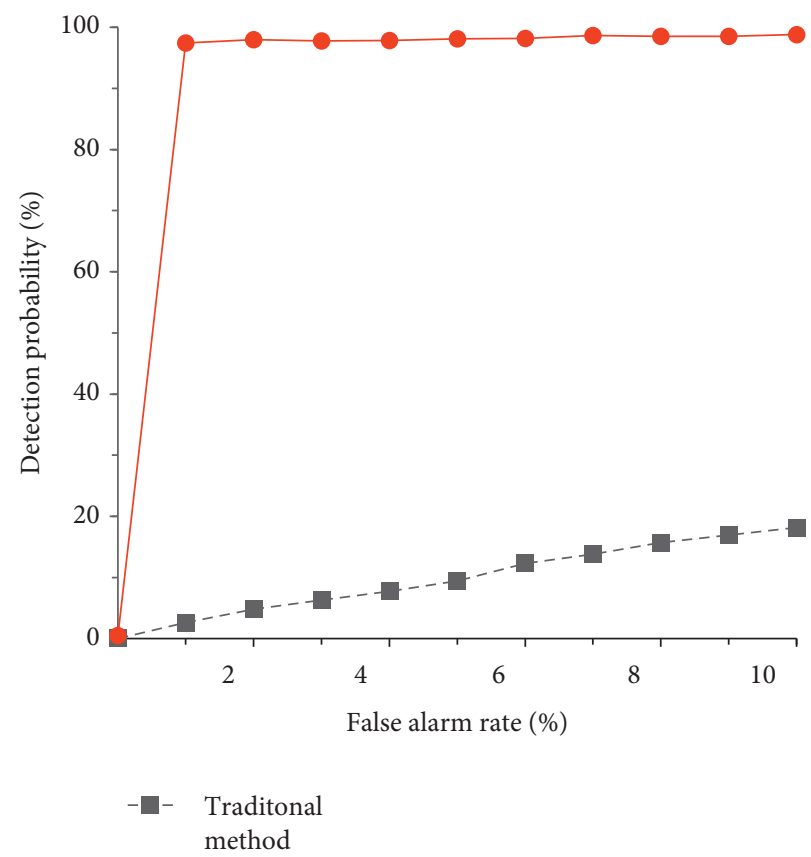

FIGURE 7: Detection rate of wavelet energy detector and traditional energy detector.

\section{Conclusion}

Currently, the accuracy of intelligent AS needs further improvement. Under the background of the rapid development of intelligent FOS technology, this paper studies the signal detection of security systems based on the feature extraction, recognition, and detection of intelligent FOS technology. Then, to improve the detection probability, this paper introduces the research background, implements the double M-Z OF-based interference sensor model, introduces the WA method, obtains the signal feature classification results, and finally, verifies the performance of the system. Specifically, this paper mainly studies the intrusion signal detection of the distributed FOS-based security system. The results show that the sensing arm and reference arm of the distributed OF-based perimeter security system can be laid in the same OC. The signal $\mathrm{F}$ of the four kinds of noise reads as follows: the intruder knocking-induced signal $\mathrm{F}$ is $0-250 \mathrm{kHz}$, the intruder climbing-induced signal $\mathrm{F}$ is $0-25 \mathrm{kHz}$, the noiseless environment-induced signal $\mathrm{F}$ is $0-1.5 \mathrm{kHz}$, and the rainy environment-induced signal $\mathrm{F}$ is $0-3.5 \mathrm{kHz}$. That is, the four kinds of environmental noise have achieved excellent results after wavelet threshold denoising. Afterward, the received signal is decomposed into seven layers by multiscale WA theory. The signal feature classification is based on WA and characterized by variance. The classification results of signal features in the four actual situations can greatly improve the system's performance. The detection probability of the system is $99 \%$. The intelligent FOS system based on WA has excellent signal detection performance. The research content provides a certain reference for the application of intelligent FOS technology in the security system. However, some deficiencies have to be pointed out. Firstly, this paper does not conduct in-depth analysis and research on the environmental background noise along with the OC, such as the phase modulationinduced random phase drift of the thermal expansion of the OF caused by the environmental temperature change and the noise caused by the random polarization state change of the single-mode OF. Secondly, there is no in-depth exploration of feature extraction and new method recognition of various detection signals along the OF. In the later research, the OF performance-induced noise will be studied combined with the influencing factors, such as the temperature of the $\mathrm{OF}$, and more new detection signal feature extraction methods will be explored. 


\section{Data Availability}

The smart sensor data used to support the findings of this study are included within the article.

\section{Conflicts of Interest}

The authors declare that they have no conflicts of interest regarding the publication of this study.

\section{References}

[1] C. G. Berrocal, I. Fernandez, and R. Rempling, "Crack monitoring in reinforced concrete beams by distributed optical fiber sensors," Structure and Infrastructure Engineering, vol. 17, no. 1, pp. 124-139, 2021.

[2] X. Chapeleau and A. Bassil, "A general solution to determine strain profile in the core of distributed fiber optic sensors under any arbitrary Strain fields," Sensors, vol. 21, no. 16, Article ID 5423, 2021.

[3] M. Lakomski, G. Tosik, and P. Niedzielski, "Optical fiber sensor for PVC sheet piles monitoring," Electronics, vol. 10, no. 13, Article ID 1604, 2021.

[4] S. T. Kim, H. Yoon, Y.-H. Park, S.-S. Jin, S. Shin, and S.-M. Yoon, "Smart sensing of PSC girders using a PC strand with a built-in optical fiber sensor," Applied Sciences, vol. 11, no. 1, Article ID 359, 2021.

[5] F. Asriani, G. Winasis, and G. Pamudji, "Sensitivity of optical fiber sensors to deflection of reinforced concrete beam," IOP Conference Series: Materials Science and Engineering, vol. 982, no. 1, Article ID 012025, 2020.

[6] A. T. Kurzych, L. R. Jaroszewicz, M. Dudek et al., "Measurements of rotational events generated by artificial explosions and external excitations using the optical fiber sensors network," Sensors, vol. 20, no. 21, Article ID 6107, 2020.

[7] P. Lu, L. Ye, Y. Zhao, B. Dai, M. Pei, and Z. Li, "Feature extraction of meteorological factors for wind power prediction based on variable weight combined method," Renewable Energy, vol. 179, pp. 1925-1939, 2021.

[8] R. Al-Ruzouq, M. B. A. Gibril, A. Shanableh et al., "Sensors, features, and machine learning for oil spill detection and monitoring: a review," Remote Sensing, vol. 12, no. 20, Article ID 3338, 2020.

[9] Z. Ballard, C. Brown, A. M. Madni, and A. Ozcan, "Machine learning and computation-enabled intelligent sensor design," Nature Machine Intelligence, vol. 3, no. 7, pp. 556-565, 2021.

[10] R. Cardona Huerta, F. Moreu, and J. A. Lozano Galant, "Aerial tramway sustainable monitoring with an outdoor lowcost efficient wireless intelligent sensor," Sustainability, vol. 13, no. 11, Article ID 6340, 2021.

[11] M. Mounir, E. Adil, C. Nabil, and I. Mohamed, "The use of magnetostrictive intelligent sensors for fault tolerant control of induction motors with energy harvesting principle," Transactions of the Institute of Measurement and Control, vol. 43, no. 9, pp. 1996-2005, 2021.

[12] O. Ülgen, R. Shnaiderman, and C. Zakian, "Interferometric optical fiber sensor for optoacoustic endomicroscopy," Journal of Biophotonics, vol. 14, no. 7, Article ID 202000501, 2021.

[13] M. C. Alonso-Murias, D. Monzón-Hernández, O. RodríguezQuiroz et al., "Long-range multicore optical fiber displacement sensor," Optics Letters, vol. 46, no. 9, pp. 2224-2227, 2021.
[14] L. Craig, "An optical fiber sensor to measure strain in solid rocket motors," International Journal of Advancements in Technology, vol. 12, no. 1, pp. 1-7, 2021.

[15] G. Lu, T. Zhu, and Y. Xu, "Low velocity impact energy monitoring and recognition of composite laminates with variable thickness based on optical fiber sensor Network," Applied Sciences, vol. 11, no. 2, Article ID 584, 2021.

[16] R. Min, Z. Liu, L. Pereira, C. Yang, Q. Sui, and C. Marques, "Optical fiber sensing for marine environment and marine structural health monitoring: a review," Optics \& Laser Technology, vol. 140, Article ID 107082, 2021.

[17] M. F. Bado, J. R. Casas, and J. Gómez, "Post-processing algorithms for distributed optical fiber sensing in structural health monitoring applications," Structural Health Monitoring, vol. 20, no. 2, pp. 661-680, 2021.

[18] W. Du, J. Chai, D. Zhang, and W. Lei, "The study of waterresistant key strata stability detected by optic fiber sensing in shallow-buried coal seam," International Journal of Rock Mechanics and Mining Sciences, vol. 141, Article ID 104604, 2021.

[19] R. V. Mariana, F. M. Douglas, and C. S. M. Larissa, “A calibrated intelligent sensor for monitoring of particulate matter in smart cities," Sensors \& Transducers, vol. 250, no. 3, pp. 1-9, 2021.

[20] B. Marzena, T. Tomasz, and D. Jakub, "New technologies for smart cities - high-resolution air pollution maps based on intelligent sensors," Concurrency and Computation: Practice and Experience, vol. 32, no. 13, Article ID 12337, 2020.

[21] S. Jou-Claus, A. Folch, and J. Garcia-Orellana, "Applicability of Landsat 8 thermal infrared sensor for identifying submarine groundwater discharge springs in the Mediterranean Sea basin," Hydrology and Earth System Sciences, vol. 25, no. 9, pp. 4789-4805, 2021.

[22] D. A. Kozorez, D. M. Kruzhkov, and V. A. Yakimenko, "Improved processing of data from micromechanical sensors in a universal integrated navigation system," Russian Engineering Research, vol. 41, no. 8, pp. 752-755, 2021.

[23] T. Wischmeyer, J. R. Stetter, W. J. Buttner, V. Patel, and D. Peaslee, "Characterization of a selective, zero power sensor for distributed sensing of hydrogen in energy applications," International Journal of Hydrogen Energy, vol. 46, no. 61, pp. 31489-31500, 2021.

[24] W. Ibrahim Gabr, M. A. Ahmed, and O. M. Salim, "Hybrid detection algorithm for online faulty sensors identification in wireless sensor networks," IET Wireless Sensor Systems, vol. 10, no. 6, pp. 265-275, 2020.

[25] M. Nilanjan, K. P. Alak, and P. S. Satya, "Interfacial delamination in glass-fiber/polymer-foam-core sandwich composites using Singlemode-Multimode-Singlemode optical fiber sensors: identification based on experimental investigation," Journal of Sandwich Structures and Materials, vol. 22, no. 1, pp. 40-54, 2020.

[26] U. Róbert, P. Vlaho, and Y. P. Lucy, "Uncertainty identification of blade-mounted lidar-based inflow wind speed measurements for robust feedback-feedforward control synthesis," Wind Energy Science, vol. 4, no. 4, pp. 677-692, 2019.

[27] S. Gopikrishnan, P. Priakanth, and R. M. Awangga, "HSIR: hybrid architecture for sensor identification and registration for IoT applications," The Journal of Supercomputing, vol. 75, no. 8, pp. 5000-5018, 2019.

[28] A. Akshay, K. Rohit, and W. Manya, "Iris sensor identification in a multi-camera environment," Information Fusion, vol. 45, pp. 333-345, 2019. 
[29] S. U. Jan, Y. D. Lee, and I. S. Koo, "A distributed sensor-fault detection and diagnosis framework using machine learning," Information Sciences, vol. 547, pp. 777-796, 2021.

[30] A. Mohammadi and A. Ramezani, "An analytical robust sensor fault detection and fault tolerant control in quadrotor," Automatic Control and Computer Sciences, vol. 55, no. 1, pp. 38-52, 2021.

[31] A. Q. Fatima, S. Adel, and A. A. Saad, "The lightweight feature extraction method for efficient acoustic-based animal recognition in wireless acoustic sensor networks," EURASIP Journal on Wireless Communications and Networking, vol. 2020, no. 1, pp. 1-21, 2020.

[32] H. S. Fatemeh, P. Naser, and B. Mohammad, "Sensor fault detection in a class of nonlinear systems using modal Kalman filter," ISA Transactions, vol. 107, pp. 214-223, 2020.

[33] D. P. Dengping Hu, C. Guo, and X. Chu, "Nondestructive sensor detection and economic applicability analysis of underground foundation pit," Russian Journal of Nondestructive Testing, vol. 56, no. 8, pp. 681-688, 2020.

[34] J. Xu, J. Wang, Y. Tian, J. Yan, X. Li, and X. Gao, "SE-stacking: improving user purchase behavior prediction by information fusion and ensemble learning," PLoS One, vol. 15, no. 11, Article ID e0242629, 2020. 